\title{
Présentation. Redécouvrir, en Virgile, l'immense rhéteur
}

\section{Francis Goyet}

\section{(2) OpenEdition \\ 1 Journals}

Édition électronique

URL : http://journals.openedition.org/rhetorique/174

DOI : 10.4000/rhetorique. 174

ISSN : 2270-6909

Éditeur

UGA Éditions/Université Grenoble Alpes

Édition imprimée

ISBN : 978-2-84310-263-9

Référence électronique

Francis Goyet, « Présentation. Redécouvrir, en Virgile, l'immense rhéteur », Exercices de rhétorique [En ligne], 2 | 2013, mis en ligne le 28 janvier 2014, consulté le 12 septembre 2020. URL : http:// journals.openedition.org/rhetorique/174 ; DOI : https://doi.org/10.4000/rhetorique.174

Ce document a été généré automatiquement le 12 septembre 2020.

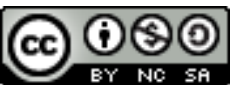

Les contenus de la revue Exercices de rhétorique sont mis à disposition selon les termes de la Licence Creative Commons Attribution - Pas d'Utilisation Commerciale - Partage dans les Mêmes Conditions 4.0 International. 


\title{
Présentation. Redécouvrir, en Virgile, l'immense rhéteur
}

\author{
Francis Goyet
}

1 À bien regarder Virgile, dit Tibère Claude Donat à son lecteur ${ }^{1}$, «tu découvriras un immense rhéteur [rhetorem summum], et tu comprendras par là qu'il aurait fallu confier Virgile non pas aux grammairiens, mais bien plutôt aux maîtres de rhétorique [oratores] ». Et d'ajouter : « C'est Virgile, en effet, qui te fera voir la plénitude de l'art de parler » : «artem dicendi plenissimam demonstrabit ». Voilà l'évidence pour l'Antiquité et l'âge classique, une évidence que nous avons redécouverte dans l'équipe RARE à partir de 1998, et que les latinistes redécouvrent eux aussi depuis la même époque. Notre dossier d'articles sur Virgile participe ainsi d'un mouvement général de réhabilitation, signifié avec éclat par Paul Veyne et sa nouvelle traduction de l'Énéide ${ }^{2}$. Ce retour d'intérêt pour l'Énéide passe, entre autres, par un retour aux rhétoriciens qui l'ont commentée. C'est assez prévisible, puisque, dans cette épopée, les discours de personnages ont une présence massive, y occupant quantitativement plus d'un tiers de l'œuvre.

2 Une telle redécouverte se heurte aujourd'hui à de puissantes idées préconçues sur Virgile. Donat lui-même était bien conscient de lutter contre le point de vue des grammairiens ou philologues. Nous avons, nous, à lutter contre l'antinomie trop aisément affirmée entre poésie et rhétorique, entre sublime et technique. Dans cette présentation, on évoquera donc moins les articles qui suivent que le contexte général dans lequel s'inscrit leur publication. Pour cela, il suffit et de raconter la manière dont l'équipe a découvert les commentaires rhétoriques sur Virgile, et de remonter aux enjeux de ces commentaires aux $\mathrm{XVI}^{\mathrm{e}}$ et $\mathrm{XVII}^{\mathrm{e}}$ siècles. Ce sont pour ainsi dire deux folies. La nôtre dans l'équipe peut se nommer la folie Ferrazzi. Mais elle découle elle-même de la folie Melanchthon. Soit une remontée de l'ordre chronologique, les années 1690 pour le premier, et 1530-1550 pour le second. 


\section{Ferrazzi, 1694}

3 Le récit de l'équipe ne peut se faire qu'à la première personne du pluriel, tant c'est, depuis le début, une aventure collective. Lors de la séance inaugurale de septembre 1998, nous avions le choix entre des commentaires ou plutôt analyses rhétoriques assez variés, sur trois sortes de discours : ceux de Cicéron, de certains historiens antiques (Tite-Live, Dion Cassius), de personnages dans l'Énéide. Suite à la discussion, c'est par ce dernier cas que nous avons décidé de commencer. Il y avait quelque chose de subtilement paradoxal, et donc de stimulant, dans l'idée de coupler analyse rhétorique et texte poétique. L'Énéide nous parlait plus, étant plus évidemment littéraire que les discours de Cicéron. La forme générale de l'analyse proposée était attirante, très claire et systématique, peu éloignée au fond de l'explication de texte à la française. Enfin, la clarté tenait aussi à la brièveté des discours de personnages ${ }^{3}$ : ceux retenus par le commentateur sont souvent de vingt à trente vers, soit une longueur très proche des textes que nous donnons aujourd'hui à expliquer aux étudiants - alors qu'un discours de Cicéron, c'est trop long. Au total, ces analyses de l'Énéide nous faisaient une double promesse : revisiter, dans l'espoir d'accrocher vraiment, un de ces grands classiques qui n'a été lu que par devoir; prendre pour guide les pédagogues de l'époque, avec cet autre espoir, de saisir l'esprit même de leur pédagogie.

4 À l'époque, nous ignorions à peu près tout de la masse des commentaires rhétoriques de l'âge classique sur Virgile. Nous ignorions également tout du contexte qui avait vu naître ce commentaire précis de l'Énéide, ces Exercitationes rhetoricae dont nous avons tiré le nom même de la présente revue, Exercices de rhétorique ${ }^{4}$. Dans l'exemplaire de la B. M. de Grenoble, l'opuscule se trouve à la fin du volume des Euvres de Virgile publié en Allemagne (Munich et Ingolstadt, Crätz, 1760), volume qui est une des nombreuses reprises de l'édition Ad usum Delphini de Virgile donnée en 1675 par le jésuite Charles La Rue, professeur de rhétorique à Louis-le-Grand en 1677-1678 puis prédicateur célèbre ${ }^{5}$. Les Exercitationes sont donc, encore aujourd'hui, attribuées à tort à La Rue dans les catalogues de bibliothèque et sur Google ${ }^{6}$. Crätz étant l'éditeur attitré des jésuites de Bavière, et La Rue appartenant à la Compagnie, notre première idée fut que tout cela relevait de la pédagogie jésuite.

5 C'est en lisant ensemble l'ouvrage que nous sommes remontés au véritable auteur. En effet, l'un des grands intérêts des Exercitationes fut d'emblée à nos yeux leurs renvois très précis à la Rhétorique d'Aristote, et en particulier aux motifs ou «lieux» des passions de son livre II. Mais le système de référençage adopté ne correspondait à aucune édition connue d'Aristote. Le premier catalogue informatisé de la BnF nous a heureusement mis sur la voie, en nous donnant une autre occurrence des deux mots du titre, Exercitationes rhetoricae, cette fois pour l'analyse de cent quatre-vingt discours chez Tite-Live: Padoue, "Typis Seminarii ", "Apud Joannem Manfrè », 1740 pour l'exemplaire de la $\mathrm{BnF}^{7}$, même présentation générale et même type de renvois à Aristote. Nous avons ensuite trouvé assez vite l'édition de référence de la Rhétorique, également publiée, en 1689, aux Presses du Seminario ${ }^{8}$. Même si les Exercitationes sur Tite-Live comme sur Virgile sont anonymes, nous savions dès lors que leur auteur avait peu de chance d'être le très parisien La Rue, si leur milieu de production était le Séminaire de Padoue.

6 L'historique du Séminaire nous a donné le fin mot de l'enquête. Dès avant 1670, l'institution est dirigée par le cardinal Gregorio Barbarigo (1625-1697), personnage très 
important qui fut un papabile et a ensuite été canonisé. Le cardinal a dès le début un intérêt puissant pour la rhétorique, qui lui parait indispensable pour la formation des futurs prêtres9. L'un de ses gestes de rénovateur des études est la fondation en 1680 des Presses du Séminaire, qu'il confie à Giovanni Manfrè. Un autre geste est de confier la charge-clé de préfet des études à un jeune homme de moins de trente ans, et qui s'avère être l'auteur des Exercitationes. Son nom est, selon la graphie vénitienne, Marco Antonio Ferrazzi (1661-1748), en latin Ferraccius, Ferratius, etc. Repéré à treize ans par le cardinal, il est nommé professeur d'hébreu en 1683, à vingt-deux ans, puis préfet des études en 1687 ou $1688^{10}$. Avec cette nouvelle fonction, le cardinal l'a clairement chargé de réformer et dynamiser le cursus, et c'est mû par cette ambition que Ferrazzi rédige alors les deux Exercitationes. Mais son zèle de réformateur est tel que ses collègues, excédés, obtiennent sa disgrâce en 1692 - il est nommé chanoine à Monselice. La disgrâce est sans doute l'une des raisons de l'anonymat de ses deux Exercitationes : il y a eu effacement du nom, damnatio memoriae. L'autre raison est l'usage de l'époque: l'anonymat est une façon de dire que les ouvrages sont une commande de l'institution. Comme le dira bien plus tard Ferrazzi, en 1729, le véritable auteur ou plutôt «auctor » de l'entreprise, ce fut dès le départ le cardinal Barbarigo. Ce fut lui en tout cas qui, au vu des Exercitationes sur Tite-Live, avait demandé à Ferrazzi de faire de même sur tout Cicéron, avec le conseil d'ajouter le texte des discours pour que les analyses soient plus lisibles $^{11}$.

7 De la redécouverte de Ferrazzi, nous pouvons déjà tirer une conclusion, sur l'idée de « scolaire » à l'âge classique.

8 Si on se place du point de vue des historiens de l'éducation, ce que l'équipe a retrouvé est très banal. C'est un phénomène bien connu que cette symbiose entre ouvrages pour la classe et institutions éducatives de niveau universitaire, ou pré-universitaire (la classe de rhétorique, qui correspondait à l'actuelle $1^{\mathrm{e}}$ française, relevait en général de l' Academia, équivalent du moderne college anglo-saxon). En faisant l'histoire d'un livre, on restitue en fait celle de l'institution. C'est un gain, puisque les ouvrages « utilitaires » de ce genre sont désormais sous-estimés, et leurs auteurs, voués à l'oubli, surtout lorsque, comme ici, les usages de l'institution leur ont même interdit de signer l'ouvrage.

9 Le résultat est moins banal du point de vue de l'histoire de la rhétorique, même s'il est là aussi récurrent. Le «scolaire " n'est pas plat, mais tendu par un but de rénovation. Derrière l'inconnu Ferrazzi, nous entrevoyons un puissant mouvement de réforme des études, porté de bout en bout par un cardinal d'envergure, qui sera canonisé et dont le modèle est à l'évidence Charles Borromée, le saint évêque de Milan au tournant des XVI et XVII ${ }^{e}$ siècles. Le cardinal Barbarigo est très attentif à son Séminaire, qu'il appelle l'âme de son âme, ou plus exactement « le cœur de son cœur ${ }^{12}$ ». La rhétorique est donc le cœur du cœur de son cœur, l'élément fondamental d'une préparation à la prêtrise.

10 L'ambition réformatrice de Barbarigo est ce qui motive à son tour la folie Ferrazzi. Le zèle de ce dernier pour la rhétorique est de même un zèle de réformateur. Car il faut du zèle pour se lancer dans l'analyse rhétorique minutieuse et systématique de tous les discours de Tite-Live puis de Virgile. Du zèle, ou de la folie, à soulever les montagnes. La foi dans la pédagogie est souvent, à l'époque, adossée à une foi religieuse. Cette configuration est très familière. Avant Ferrazzi, le descriptif est le même, on le sait, pour les jésuites de l'âge classique, aussi ardemment cicéroniens que catholiques. Il est le même aussi pour Christoph Schrader (1601-1680), professeur de rhétorique à 
l'Université de Helmstedt et une sorte d'inspecteur général ou de ministre de l'éducation, la main dans la main avec le duc Auguste ${ }^{13}$. Schrader, élève de Vossius, est comme son maître un militant du retour à Aristote, ce dont Ferrazzi s'inspire sans aucun doute ${ }^{14}$. Vossius à son tour n'est pas seulement un savant à la renommée européenne, il est aussi un acteur de premier plan dans la réforme des études aux PaysBas. Enfin, l'un des inspirateurs de Vossius, Melchior Junius (1545-1604) est de même un personnage de premier plan, professeur de rhétorique et à la fois recteur de l'Academia de Strasbourg, où il succède à son maitre le célèbre Sturm, lequel est aussi le maître du célébrissime Ramus. On pourrait multiplier les exemples.

11 Tous ces savants font preuve, comme enseignants, d'une activité stupéfiante, avec une confiance à toute épreuve dans la valeur de ce qu'ils transmettent, une foi pour ainsi dire militante. On peut se moquer, et conclure que la réforme des programmes est décidément la maladie du pédagogue, à l'âge classique comme au nôtre. Mais appeler cela « scolaire ", c'est être bien peu sensible et à l'envergure de ces entreprises, et à la foi qui les anime. Pour retrouver Virgile avec les rhéteurs, il nous faut d'abord dépasser notre préjugé contre l'ancien monde scolaire, qu'il vaudrait mieux dire en l'occurrence universitaire $^{15}$.

\section{Melanchthon, 1530}

Si nous poursuivons à partir de Junius la remontée dans le temps, on trouve la source d'où tout découle, et qui est l'idéal-type de pareille ambition, folie ou foi : c'est la figure de Melanchthon - chez qui la mère de Junius a été élevée. Successeur de Luther à l'Université de Wittenberg, Melanchthon est à la fois, et par excellence, pour toute l'Europe, le Réformateur religieux et le réformateur pédagogique. Il est, en particulier, le réformateur de l'enseignement de la rhétorique, l'une de ses disciplines phares. C'est Melanchthon qui transforme en impératif le vœu de Rudolf Agricola d'en passer par les exercices, et donc par cette explication minutieuse des discours que Ramus puis les jésuites ont adoptée et dont Ferrazzi est l'un des représentants parmi bien d'autres, plus ou moins connus.

En fait de rhétorique, Melanchthon a eu comme première urgence de dégager le plan et une analyse détaillés de tous les discours et lettres de Cicéron. En étudiant la dispositio, l'économie de chaque discours, on donne à admirer les grandes beautés de ce que Melanchthon nomme la compositio ciceroniana, c'est-à-dire la manière dont Cicéron a écrit ou plutôt «composé » ses discours. L'analyse du produit fini (de la compositio) a pour but de remonter à la compréhension du grand art qui l'a produit (la dispositio). Mais Cicéron n'était qu'un début. Melanchthon a eu d'emblée le vaste programme de faire de même pour tous les discours qui se trouvent chez les principaux auteurs de l'Antiquité, ce qui inclut les historiens et les poètes (tragédie, comédie, poésie lyrique), sans oublier la Bible (à commencer par les Psaumes). Ce programme, relancé peu après par Ramus, a été largement réalisé pendant les deux siècles suivants, surtout dans l'aire germanique ${ }^{16}$.

14 Pour ce qui est de Virgile lui-même, Melanchthon a simplement publié ses œuvres en 1530, en ajoutant, dans les marges, des notes très brèves sous forme de manchettes en face des vers concernés. Lui-même travaille avec sous la main le commentaire de Landino, dont il synthétise ce qui relève de l'analyse rhétorique - Landino ayant surtout pour objectif l'allégorisation chrétienne de Virgile. Mais après Melanchthon, les 
commentaires proprement rhétoriques de Virgile abondent. On trouvera dans la bibliographie un essai de liste cumulative, à partir des divers auteurs mentionnés par les articles qui suivent.

De façon générale, les commentateurs ont un trait caractéristique commun. À partir de Melanchthon, le geste de base de tout rhétoricien va être un geste de découpage qui est d'abord mental. Il s'agit de tirer un trait, une ligne de démarcation, pour isoler les parties du discours, puis les sous-parties, etc. Ce faisant, on dégage la dispositio, qu'on l'appelle plan ou structure d'ensemble. C'est cela le point crucial, l'enjeu qui synthétise tous les résultats partiels de l'analyse et leur donne sens. Melanchthon l'a imposé comme une exigence standard, le prérequis de toute analyse rhétorique sérieuse, et c'est un des fondamentaux de sa méthode pédagogique. On le voit bien à propos de Virgile. Le premier commentateur (sauf oubli) à donner systématiquement le plan des discours dans l'Énéide est de façon symptomatique un des meilleurs élèves de Melanchthon, Strigel - le maître lui-même n'ayant pas pu, dans ses notes marginales à Virgile, donner à proprement parler le plan.

La question pour nous est de savoir pourquoi il est si important de dégager les parties et ainsi la dispositio d'ensemble. Quand on consulte les traités de rhétorique de l'Antiquité, on a le sentiment que le plan est assez routinier et ne pose pas de problème particulier : exorde, narration, argumentation, réfutation, péroraison. Mais d'une part, il s'agit là pour l'essentiel du plan d'une plaidoirie, on est dans la rhétorique des avocats. D'autre part, dès que l'on s'aventure, avec les historiens et les poètes, dans un monde où les discours sont plus diversifiés qu'au tribunal, le plan "canonique " se brouille et n'a pas cette belle simplicité. Enfin et surtout, pour les avocats comme pour les autres, les traités sont en réalité peu diserts sur la dispositio. Ils répètent que c'est à chaque orateur de juger de l'ordre dans lequel mettre ses arguments, d'user ou non de circonlocutions dans l'exorde, d'être ou non violemment pathétique dans la péroraison. Les traités renvoient donc toutes ces décisions fondamentales à la sagesse pratique de l'orateur (sa prudentia) : à son sens tout militaire de la stratégie et de la tactique, de l'adéquation au terrain, au « ici et maintenant ».

Or, autant de discours, autant de situations singulières. Si l'exploration tous azimuts de Melanchthon est animée par le désir un peu vertigineux d'étudier chez tous les auteurs la dispositio sur pièces, c'est parce qu'il n'y a pas moyen de faire autrement. Sa folie est fondée en raison: l'art rhétorique est, comme l'art militaire, un art d'application. Melanchthon ne se tourne pas vers la rhétorique appliquée pour être concret, pour illustrer la théorie, sortir de l'abstraction, bref, se mettre à la portée des élèves. Non : la rhétorique appliquée, pratique, lui permet de combler le silence des traités sur la dispositio, un silence retentissant, puisqu'il s'y joue apparemment l'essentiel. Melanchthon impose donc de s'exercer à la rhétorique, parce que les exercices sont le seul moyen de parvenir à comprendre le génie pour ainsi dire militaire de chaque orateur, de saisir comment, en situation, il a emporté la bataille. Le but des exercices est d'explorer les divers possibles en fait de dispositio, de plan ou plutôt d'économie du discours. Il ne s'agit pas d'exclure la théorie, mais de la compléter par la "pratique ", qui se dit en latin usus ou exercitatio. Chez les jésuites, le règlement des études (Ratio studiorum) le dit à sa manière, en précisant qu'en classe de rhétorique le professeur consacrera chaque jour une demi-journée à la théorie, l'autre à la «pratique » ou usus : façon pédagogique de dire la complémentarité des deux. Le va-et-vient entre théorie et pratique est structurant. 
Chez Melanchthon et pendant tout l'âge classique, le mot qui résume cette ambition de comprendre est donc celui d'usus et son synonyme exercitatio : la " pratique ", la mise en usage, le passage à l'acte, l'expérimentation. L'erreur à ne pas faire serait de croire que "pratique » ici signifie seulement : pratique d'écriture puis de déclamation. C'est assurément l'un des sens du mot, et de fait toute la pédagogie du temps en vient là, et ce, jusqu'à la fin du xix ${ }^{e}$ siècle. La finalité ultime était bien que l'élève à un moment donné prépare lui-même un discours, et montre ainsi ce qu'il a appris à la lecture des grands orateurs. Mais « pratique » signifiait aussi, et même principalement au cours de l'année, l'analyse des discours, que ce soit ceux des orateurs réels comme Cicéron, ou ceux des personnages fictifs chez Tite-Live ou Virgile. Si l'élève apprend à la lecture des discours, c'est qu'il s'agit d'une lecture pour ainsi dire active, éclairée par l'analyse rhétorique sous la direction d'un maître.

19 L'un des équivalents grecs d'exercitatio étant askèsis, l'«ascèse " ou plutôt l'entraînement intensif, tendu vers l'excellence du résultat, la comparaison ici la plus éclairante est celle avec le monde des athlètes ${ }^{17}$. Le professeur de rhétorique était dans la situation qui est celle, aujourd'hui, du coach des sportifs de haut niveau. Ceux-ci ont les deux mêmes sortes d'usus : pratiquer leur sport (préparer et dire des discours) mais aussi en analyser toutes les composantes, ou bien de façon théorique, avec par exemple des cours d'anatomie et de diététique (les traités), ou bien de façon pratique, en visionnant avec le coach la vidéo d'autres sportifs en pleine action (lire et analyser des discours). Lire et analyser, c'est donc bien s'exercer. Cela fait partie intégrante de la pratique d'un sport, comme de la pratique de la rhétorique. Sans cela, on ne progressera pas dans la discipline choisie. Pour reprendre la définition de Tesmar, l' usus est une " ratio imitandi » : une méthode pour faire aussi bien que les prédécesseurs, voire mieux ${ }^{18}$. Voilà tout ce que comporte le titre Exercices de rhétorique.

Le titre de Ferrazzi en 1694 est ainsi le lointain écho des ambitions de Melanchthon, Sturm et Ramus au XVI ${ }^{e}$ siècle. La théorie et les traités ne suffisent pas, il faut montrer comment la rhétorique fonctionne, en pratique, à même les textes, si l'on veut que les étudiants puissent la pratiquer à leur tour. Le sens du travail des rhétoriciens pendant tout l'âge classique est de faire de ce rêve une réalité. Le programme de travail est un véritable défi, qui rejoint à sa manière celui en 1550 de la Défense et illustration de la langue française. Du Bellay rêve d'un orateur qui en prose nous émouvrait autant que Cicéron, et d'un poète à venir capable de nous faire vibrer en français avec des vers aussi beaux que ceux de Virgile, et qui serait proprement un «Virgile français ${ }^{19}$ ». Or le programme a été tenu. Le Virgile français, c'est Racine, qui n'est pas précisément un inculte en matière de rhétorique.

21 Pour conclure sur la folie Melanchthon, qui est aussi celle de Ramus, le découpage d'un discours en parties engage l'essentiel, à savoir la compréhension de la dispositio. Les rhétoriciens de l'époque ont donc trouvé un mot qui veut dire « découpage » en grec, mais est nettement plus noble, celui d'analyse, qu'ils nomment dans leur latin analysis ou resolutio. Ramus dédouble même le mot grec en "analyse ou diérèse ", avec en miroir "genèse ou synthèse ", mots repris d'Aristote ${ }^{20}$. Ce sont pour lui les «deux manières d'exercice ", c'est-à-dire d'usus ou exercitatio. L'« analyse ou diérèse » consiste à tirer un trait, à découper un texte, à le décomposer en éléments et les identifier, sous la direction d'un maître ${ }^{21}$. La "genèse ou synthèse » est le moment où l'on compose soimême un autre texte, le latin compositio étant formé comme le grec sunthesis. Les deux voies s'appellent l'une l'autre. Chez Ramus et d'abord chez Melanchthon, elles sont le 
fondement même de leur pédagogie, comme de celle des jésuites en classe de rhétorique. Pas d' "analyse " sans "synthèse ", et réciproquement: voilà l'enjeu de l' usus. Comment apprendre à écrire aussi bien que les grands maîtres d'autrefois, en prose ou en vers ? La réponse est : en « analysant » leurs écrits.

Il faut laisser les guillemets, car analyser un texte, aujourd'hui, est et n'est pas la même chose. Cela désigne seulement un art de lire, lequel n'est pas traversé par la finalité d'écrire à son tour ${ }^{22}$. De nouveau, on peut se moquer, et trouver cocasse qu'à l'école, encore au XIX ${ }^{e}$ siècle, le maître fasse rédiger des narrations à un Baudelaire ou à un Flaubert, et des discours à un Sainte-Beuve ou à un Victor Cousin. Le point est que l'analyse des récits des grands auteurs est très différente s'il s'agit de devoir composer un récit à son tour : alors on lit pour apprendre les « secrets de l'art d'écrire ${ }^{23}$ ", ce qui reformule la définition de l'usus comme ratio imitandi. Si nous ne comprenons plus l'esprit de l'ancienne pédagogie des lettres, c'est que la finalité de la nôtre n'est plus la même.

Pour conclure plus généralement, on peut essayer de deviner où va la réinvention actuelle de Virgile.

J'ai donc raconté une découverte de terrain faite en équipe. Ce fut d'abord une intuition, aussi confuse que têtue. Puis, en revisitant l'Énéide, nous nous sommes retrouvés à revisiter la rhétorique ancienne et, pour ainsi dire, nous n'en sommes pas revenus. Dans cette forêt profonde qu'est la sylve ou jungle des commentateurs, avec Virgile nous allons un peu moins obscurs et un peu moins solitaires. Le retour d'intérêt pour l'Énéide est sans doute, comme tout mouvement de réhabilitation, la confluence d'intuitions et d'expériences du même ordre.

Après l'âge classique, la rhétorique puis Virgile sont tombés aussi bas qu'ils avaient été au plus haut. La première est désormais soupçonnée d'être bêtement scolaire, et le second, tristement classique ou, si on comprend bien Florence Dupont, superficiellement néo-classique ${ }^{24}$. L'intuition vécue par les membres de l'équipe RARE, et vérifiée par tant de lectures en commun, est tout autre, et pour ainsi dire en deçà de l'interprétation. L'intuition est que la rhétorique appliquée est une des voies d'accès aux beautés mystérieuses d'un tel poète, et par là de la poésie.

Comme chez Melanchthon et les anciens rhétoriciens, la voie passe nécessairement par la dispositio. De façon frappante, chacun des articles qui suit se bat avec le plan, ou, dès que l'on regarde plusieurs commentateurs, les plans possibles, pour un seul et même discours. D'ailleurs, chacun des articles tâtonne du simple point de vue typographique : vaut-il mieux un tableau, une liste, des accolades, ou même une phrase synthétique ? Et quelle sorte de tableau faut-il pour comparer plusieurs plans, en faire saisir les différences? C'est un signe parmi d'autres que nous ne sommes qu'au début d'un long processus de réappropriation. L'art de la dispositio est le grand oublié et des traités antiques et de la critique moderne, alors même qu'il est essentiel. On ne le retrouvera pas d'un seul coup. Entre Antiquité et modernité, la leçon de l'âge classique est que la méthode passe par les exercices, par la "pratique », par la lecture lente et attentive d'un très grand nombre de textes, parce qu'il n'y a pas moyen de faire autrement. Pour réapprendre l'intérêt de la dispositio, il faut voir d'un œil critique une foule de textes ou dispositiones. C'est une folie selon le monde, c'est-à-dire en fait une sagesse. Car c'est une voie, aussi sûre qu'elle est longue. 
attendant, sous le poète, on peut déjà réapprendre à voir un rhéteur à son sommet. In poeta rhetor summus. Ou plutôt, Virgile est, dans le même mouvement, immense rhéteur et immense poète.

\section{BIBLIOGRAPHIE}

Pour la bibliographie virgilienne à l'âge classique, voir :

- Virgil in Late Antiquity, the Middle Ages and the Renaissance : An Online Bibliography

- le projet Vergils Aeneis 1502-1840 de la Bibliothèque de Munich (BSB, en ligne)

- Dolores JULIA-PRALON, notice sur le Virgile de La Rue dans M. Furno dir., La collection ad usum Delphini vol. II, Grenoble, Ellug, 2005, p. 95-122

- Ludivine Goupillaud, De l'or de Virgile aux ors de Versailles. Métamorphoses de l'épopée dans la seconde moitié du XVII siècle en France, Genève, Droz, 2005 (p. 177-188 sur La Rue)

- Craig KALLENDORF, The Virgilian Tradition : Book History and the History of Reading in Early Modern Europe, Aldershot, Ashgate, 2007 ; The Other Virgil : Pessimistic Readings of the Aeneid in Early Modern Culture, Oxford, Oxford University Press, 2007

La liste donnée ci-après, par ordre chronologique, est celle des commentateurs rhétoriques de Virgile. Une telle liste ne saurait être qu'indicative. Il suffit pour s'en convaincre de voir la longue série de noms que donne Michel de Marolles à la suite de sa traduction (L'Énéide, Paris, Guillaume de Luyne, 1662, p. 257-258, au début de ses « Remarques sur l'Eneide de Virgile »; liste signalée par Florence de Caigny). Il mêle philologues et rhétoriciens, c'est-à-dire ceux qui ont écrit « sous le nom de Commentaires, d'Annotations, de Notes, d'Animaduersions \& d'Obseruations » :

- « [...] les uns anciens, comme Servius Honoratus, Donat, Pomponius Sabinus, le Grammairien Probus, Philargyrius, Modestinus. Les autres modernes, tels que Germanus Valens, Adrian Turnebus, Louys Lucreda [La Cerda], Jacques Pontanus, Hortensius Montfortiensis, Nascimbenus, Nannius, à Myen, Abramus, Iodocus Badius Ascentius, Philippe Beroalde, Philippe Melancton, Mancinellus, Domitius Calderinus, Augustinus Dathus, Pierius Valerianus, Ianus Parrasius, Ianus Guilielmus, Baptista Pius, Robortellus, Alexandre de Naples, Chrystophorus Landinus, Ioannes Hartungus, Iodocus Vvillichius, Camerarius, Georgius Fabricius, Corrardus [Corrado], Erythreus, De Bonfinis, Iean Louys Viues, Adrianus Burlandus, Casaubon, Lipse, Marc-Antoine Muret, Scaliger, Lambin, Gifarius, Taubmannus, Claude Saulmaise, Ludouicus Lucius Professeur de Basle, Magius, Merula, Thomas Farnabius, \& Cornelius Schreuelius. »

Pontanus en 1599 (p. 20 du pdf indiqué plus bas) a une liste plus courte d'« Enarratores et Scholiastae» - en italiques les oublis de Marolles :

- « Donatus, Seruius, German. Valens, Nascimbaenus, Montfortius, Iunius Philargyr., Sebast. Corrad, Sebast. Regulus, Ramus, Bersmanus, Vrsinus, Erythraeus, Frischlinus. »

J'ajoute dans la liste ci-dessous un renvoi à Landino, Strigel et Corrado, qui ne sont pas exploités par les articles de ce Dossier, mais auxquels je me suis référé dans deux articles à paraître : Francis Goyet, « Mythologies de la préméditation et mythologies de l'improvisation : sur quelques commentaires rhétoriques de l'Énéide », Sur des vers de Virgile. Commenter l'Énéide, dir. Ch. Noille, Paris, Garnier ; «La rhétorique de Didon dans l'acte II : l'exprobratio au sein des vers 851-952 », Lectures de Jodelle, éd. E. Buron et O. Halévy, Rennes, P. U. R.

- IV siècle ap.J.-C. 
SERVIUS, P. Virgilii Maronis Opera. Mauri Servii Honorati..., Paris, Robert Estienne, 1532 (Google). L'éd. G. Thilo et H. Hagen, In Vergilii carmina commentarii, Leipzig, Teubner, 1923 (repr. de 1881; archive.org) signale par des italiques les ajouts du « Servius de Daniel » ou « Servius augmenté » (Serv. auct.), qui est parfois considéré comme une source de Ti. Donat et dont le texte a été publié pour la première fois en 1600 par Pierre Daniel (1530-1603), Pub. Virgilii [...] Aeneidos [...], Paris, Séb. Nivelle.

DONAT (Tiberius Claudius Donatus), Interpretationes vergilianae, éd. H. Georgii, Leipzig, Teubner, 1905 ; openlibrary.org.

- 1492

LANDINO, Cristoforo (1424-1504), In P. Vergilii Interpretationes, Nürenberg, Koberger, 1492 ;

diglib.hab.de. Une édition en 1507 a moins d'abréviations : Publii Vergilii Bvcolica [...]. Ad Hos Donati Fragmenta, Christophori Landini..., Venise, 1507 ; BSB00010391.

- 1530

MELANCHTHON, Philipp (1497-1560), Virgilius cum Phil. Melanchthonis scholiis, Hagueneau, Secerius, 1530 ; sous le titre Enarratio Aeneidos Virgilii dans les Opera de Melanchthon, vol. XIX, éd. K. G. Bretschneider et H. E. Bindseil, Brunswick, Schwetschke et fils (Corpus Reformatorum), 1853.

- 1553

STRIGEL, Victorinus (1524-1569), In Virgilii sex priores libros Aeneidos nouus commentarius ex privatis lectionibus, Wittenberg, sans nom d'éditeur, 1588 ; annoncé sur Google (BSB, cote 04/1 N II 623). Cours dicté de 1552 à 1555 , et édité par Stephano Riccio.

$\cdot 1555$

CORRADO, Sebastiano (mort en 1556), Commentarius, in quo P. Virgilij Maronis liber primus Aeneidos explicatur, Florence, Laur. Torrentinus, 1555 ; Google.

- 1559

HORTENSIUS MONTFORTIUS, Lambertus (Lambert van der Hove, ca. 1500-1574, que Pontanus appelle « Montfortius », et La Cerda, « Hortensius »), Enarrationes in sex priores libros Aeneidos..., Bâle, H. Petrus et Oporinus, 1559 ; Google. Pour l'éd. de 1577, voir Nascimbeni.

- 1561 SCALIGER, Jules-César (1484-1558), Poetices libri septem, Paris, Vincent, 1561, III, 24 (« Oratio »), p. 108C-113A ; Google. Texte latin et trad. all. dans l'éd. L. Deitz, vol. 2, Stuttgart-Bad Cannstatt, Frommann-Holzboog, 1994, où c'est le chap. 23.

- 1577

NASCIMBENI, Nascimbene (né à Ferrare, actif dans les années 1555-1577), Lamberti Hortensii Montfortii enarrationes [...] in XII libros P. Virgilii Maronis Aeneidos. His accessit Nascimbaeni Nascimbaenii [...] in sex primos Aeneidos libros erudita et perelegans explanatio, Bâle, H. Petrus, 1577 ; Google.

-1599

PONTANUS, Jacobus (Jacob Spannmüller, 1542-1626, sj), Symbolarum libri XVII quibus P. Virgilii Maronis Bucolica, Georgica, Aeneis..., Augsburg, Praetorius, 1599 ; Google.

- 1610

LAUBANUS, Melchior (1567-1633), Archetyporum analyticorum Aeneidem libri septem, Legnica (en allemand Liegnitz), Sartorius, 1610 ; staatsbibliothek-berlin.de.

- 1612

LA CERDA, Juan Luis de (1560-1643, sj), P. Virgilii Maronis libri sex priores Aeneidos, argumentis, explicationibus et notis illustrata, Cologne, Kinchius, 1663 ( $1^{\text {re }}$ éd. Lyon, Cardon, 1612) ; uni- 
goettingen.de. Chaque section est suivie d'un bref argumentum (résumé du plan), de l'explicatio ou commentaire rhétorique, enfin de notes empruntant beaucoup à Pontanus. Les explicationes seules sont éditées par Nicolas-Éloi Lemaire (1767-1832), dans Virgile Quae extant opera omnia..., Paris, P. Didot l'aîné, 1821 (t. VI) et 1822 (t. VII) ; Google. Cette publication tardive montre l'intérêt tout particulier de ce commentaire, grandement loué par Charles Rollin, Traité des études (1726-31), III, chap. 2, « De la poésie », art. 2, § 2, 5. Comme La Cerda s'appuie beaucoup sur Donat (et Serv. auct.), on peut dire de lui ce que dit Vallat (art. cité, p. 163) de Donat : « il perçoit les enjeux et les stratégies mieux que les autres commentateurs, et se rapproche ainsi de ce que nous avons développé depuis quelques décennies sous l'appellation d'interactions verbales. Il en analyse la psychologie et prend grand soin de mettre en évidence les rapports de force qui s'y cachent, ainsi que les problèmes de "face" ".

$\cdot 1632$

ABRAM, Nicolas (1589-1655, sj), Commentarii in Pub. Virgilii Maronis Aeneidem, Pont-à-Mousson, G. Bernard, 1632 ; Google a la $2^{\mathrm{e}}$ partie de l'éd. 1637 (sur Aen., livres VII-XII).

- 1675

LA RUE, Charles (1643-1725, sj ; en latin Ruaeus), P. Virgilii Maronis Opera [...] ad usum Delphini, Paris, Simon Benard, 1675 ; Google a la rééd. 1682, et Philadelphie, Carey et Lea, 1823). Les notes en bas de page ont parfois des remarques rhétoriques.

$\cdot 1694$

FERRAZZI, Marco Antonio (1661-1748), Exercitationes rhetoricae in praecipuas P. Virgilii Maronis orationes, quae in Aeneidum libris leguntur, Padoue, Presses du Séminaire [Manfrè], 1694 ; éd. numérique et trad. fr. pour les commentaires des discours d'Aen. I-VI sur le site de l'équipe RARE : <http://w3.u-grenoble3.fr/rare/spip/spip.php?article285>.

\section{NOTES}

1. «Si Maronis carmina conpetenter attenderis et eorum mentem congrue conprehenderis, inuenies in poeta rhetorem summum atque inde intelleges Vergilium non grammaticos, sed oratores praecipuos tradere debuisse." (Interpretationes Vergilianae, éd. H. Georgii, vol. I, Aeneidos libri I-VI (1905), Stuttgart, Teubner, 1969, p. 4 ; trad. Daniel Vallat, «Le commentaire de T.Claude Donat au chant 1 de l'Énéide, sa place dans les débats virgiliens et ses relations avec Servius », Eruditio Antiqua, 1, 2009, p. 155-184 [p. 157], en ligne). Donat « n'est jamais meilleur que lorsqu'il analyse les discours» (Vallat, p. 163, qui donne ensuite, p. 164, la traduction intégrale de l'analyse par Donat du discours de Junon à Éole, Aen. I, 65-75).

2. Paris, Albin Michel / Les Belles Lettres, 2012.

3. La première moitié environ des 88 discours est sur le site de l'équipe RARE, avec un essai de traduction française.

4. Jean Sturm a déjà le même titre, De Exercitationibus rhetoricis J. S. liber academicus, Strasbourg, N. Wyriot, 1575.

5. Volume que j'avais vu en participant au collectif préparant l'ouvrage La collection Ad usum Delphini, Grenoble, Ellug : dir. C. Volpilhac-Auger, vol. I, 2000 ; dir. M. Furno, vol. II, 2005.

6. Le titre général des Opera de 1760 dit seulement (je traduis et souligne) : «CEuvres de Virgile avec la paraphrase de Charles La Rue, auxquelles on a joint des exercices de rhétorique sur les principaux discours de l'Énéide " (quibus adjunguntur exercitationes rhetoricae in praecipuas Aeneidos orationes). Voici les éditions des Exercitationes rhetoricae selon Craig Kallendorf, A Bibliography... (cité dans la Bibliographie) : 1694-1695, 1695 (deux fois), 1720, 1721, 1735, 1747, 1748, 1760, 1789 et 1802 ; à quoi ajouter l'éd. de 1859 (Toulouse, Privat).

7. Exercitationes rhetoricae in orationes Titi Livii Patavini, NUMM-5849571 (et sur Google Livres). 
8. Aristote, De arte rhetorica... (Google, avec «Aristoteles" en nom d'auteur), sans nom de traducteur - c'est une reprise de la traduction de Maioragio, qui date de 1550 (le commentaire à la Rhétorique d'Aristote par Maioragio est publié la même année aux mêmes Presses).

9. Le cardinal est très influencé par le De rhetorica ecclesiastica d'Agostino Valier, Vérone, 1574, qu'il fait imprimer à Padoue en 1672 (selon Achille Olivieri, « Gregorio Barbarigo : sul de historia, ovvero sul tempo, nel secondo Seicento ", dans Gregorio Barbarigo patrizio veneto vescovo e cardinale nella tarda Controriforma (1625-1697), éd. L. Billanovich et P. Gios, Padoue, Istituto per la Storia Ecclesiastica Padovana, 1999, p. 253-294 [p. 272] - je remercie Enzo Neppi pour cette référence).

10. 1687 selon Giovanni Battista Ferrari, Vitae illustrium virorum Seminarii Patavini, Padoue, Presses du Séminaire, 1799 (Google), p. 14 de sa «Vie de Ferrazzi » (p. 12-25).

11. M. T. Ciceronis Orationum pars I, cum argumentis, animadversionibus et analysi M. Antonii Ferratii, Padoue, Manfrè, a2 $\mathrm{r}^{\circ}$; sur Google. Même à cette date, Ferrazzi n'y parle pas des Exercitationes sur Virgile. Il faut attendre Ferrari en 1799 (lui-même préfet des études au Séminaire) pour que la paternité soit reconnue : «Neque Oratorum tantum, Poetarum quoque latinorum Principem illustravit, suo tamen suppresso nomine.» (Ferrari, op. cit., p. 18). La paternité ne fait aucun doute pour Dante Nardo, p. 241 (n. 127) et p. 244 (n. 145) de G. Arnaldi et M. Pastore Stocchi, Storia della cultura veneta, Vicenza, Neri Pozza, 1985, t. 5, Il Settecento, vol. 1 - je remercie Gilles Bertrand et Piero Del Negro pour cette référence. Si la $1^{\text {re }}$ éd. des Exercitationes sur Tite-Live est anonyme (Manfrè, 1694), l'avis au lecteur donne le nom de l'auteur (exemplaire vu : B. U. de Würzburg). La date de 1694 est aussi celle qui apparaît sur la page de titre séparée des Exercitationes sur Virgile, chez Manfrè (B. N. de Vienne), à la fin d'un volume des CEuvres de Virgile dont la page de titre a 1695 (avec, après Opera, "quibus accessere Exercitationes Rhetoricae in praecipuas ejus Conciones »). Dans cette édition, le tout début de l'avis de l'imprimeur souligne qu'il a enchaîné les deux publications, Tite-Live puis Virgile: «Vix dum typis nostris emisso historicorum Principe, prodit in lucem, continuato penè labore, sumptuque, summus Poetarum Virgilius. »

12. "[A]ppellabat sui cordis cor" (Ferrari, De singulari beati Gregorii Barbadici studio et amore in Seminarium Patavinum opusculum, Padoue, Presses du Séminaire, 1798, p. 54, Google). Ferrari fait ensuite du cardinal un nouveau Charles Borromée : même quand il est mal portant, il va sans cesse au Séminaire ; sa présence assidue stimule élèves et professeurs, etc.

13. Lequel est à l'origine de l'actuelle bibliothèque de Wolfenbüttel : Jean-Luc Le Cam, Politique, contrôle et réalité scolaire en Allemagne au sortir de la guerre de Trente Ans, tome I, vol. 2 (La politique scolaire d'August Le Jeune de Brunswick-Wolfenbüttel et l'inspecteur Christoph Schrader, 1635-1666/80), Wiesbaden, Harrassowitz (Wolfenbütteler Forschungen, 66), 1996.

14. Ferrazzi écrit ses Exercitationes sur Tite-Live avec sous la main Johannes Tesmar, Exercitationum rhetoricarum libri VIII, éd. Daniel Stephanus, Amsterdam, L. et D. Elzévir, 1657, IV, p. 269 à 506 (analyse de 205 discours de Tite-Live; Google). Mais Tesmar ne fait aucun renvoi à Aristote: or, après Schrader et son De rhetoricorum Aristotelis Sententia et usu commentarius (Helmstedt, H. D. Müller, 1674), ce n'est plus possible en termes d'exigence universitaire. - Pour l'importance du mot usus dans le titre de Schrader, voir plus bas.

15. Ludivine Goupillaud reste à l'intérieur de ce préjugé quand elle décrit la manière dont La Rue analyse le premier discours de Didon à Énée (Aen. IV, 305-330 ; p. 84-85 dans De l'or de Virgile... cité dans la Bibliographie) : «Les trois grandes parties de la tirade, propositio, confirmatio et peroratio, font l'objet de subdivisions plus fines, qui ne laissent de côté aucune argutie oratoire. [...] De la prière à la menace, [...] le jésuite n'omet rien des différentes modalités du discours ; peu à peu, il transforme l'appareil critique qui entoure le texte en un manuel de rhétorique illustré: l'ars amatoria repose sur une ars dicendi finalement très académique. Cette méthode trouvera d'ailleurs son prolongement dans un cahier d'exercices publié en 1694 et maintes fois réédité.» Au contraire, pour Kallendorf, ces mêmes Exercitationes sont la chance de "recover with considerable precision how students and teachers read Virgil in the classroom ». Et d'ajouter : " these humble, unduly neglected volumes can be used to offer a commentary on some of the basic generalizations about 
Renaissance education that other scholars have proposed over the last couple of decades " ("Virgil in the Renaissance Classroom: From Toscanella's Osservationi [...] sopra l'Opere di Virgilio to the Exercitationes rhetoricae ", The Classics in the Medieval and Renaissance Classroom, éd. J. F. Ruys et al., Turnhout, Brepols, 2013, p. 309-328 [p. 317-318 et p. 321-322]).

16. Le corpus actuel de l'équipe RARE comprend ainsi des analyses rhétoriques détaillées pour la totalité des discours de Cicéron, Tite-Live, Salluste, Quinte-Curce, la Bible, et même (chez Tesmar) Marc-Antoine Muret ; la totalité de Virgile, Horace, Perse, Pindare ; un certain nombre de discours isolés (Tacite, Ovide...) et, chez Melanchthon, l'analyse rapide de pièces de théâtre (Térence).

17. Un des sens d'askèsis en grec est «l'entraînement de l'athlète ». Aristote emploie le mot au sens de « entraînement à la guerre » (Pol. VII, 14, 1333b39); dans l'Éthique à Nicomaque (I, 9, 1-3, 1099b5), il emploie askèton pour dire le troisième terme de la triade de toute éducation, en latin natura, ars et usus/exercitatio.

18. Tesmar, op. cit., p. 269, quand il présente son livre IV sur Tite-Live.

19. Défense et illustration, I, 5 .

20. Anal. prior. I, 38, 49a19, Soph. Elench. 169a25, etc. Ramus (Pierre La Ramée, 1515-1572), La Dialectique, éd. M. Dassonville, Genève, Droz, 1964, p. 157 - les p. 153-157 reproduisent les folios $68 \mathrm{r}^{\circ}-\mathrm{v}^{\circ}$ du Petit traicté qui fait suite à la Dialectique dans l'éd. posthume de 1576, Ramus lui-même donnant ici en français ce qu'il avait écrit en latin dans les Dialecticae institutiones de 1543, $\mathrm{f}^{\circ}$ 55-56, ou dans la Scholae de 1569. Voir Kees Meerhoff et Jean-Claude Moisan, « Précepte et usage : un commentaire ramiste de la $4^{\mathrm{e}}$ Philippique ", Autour de Ramus, texte, théorie, commentaire, éd. K. Meerhoff et J.-Cl. Moisan, Québec, Nuit blanche, 1997, p. 305-370 : aux p. 316 et p. 360, citation du texte français et latin de Ramus - tout l'ouvrage, par ses nombreuses études d'analyses ramistes, concerne le propos de ma présentation; voir aussi, dans l'œuvre de Walter J. Ong, son article en français «Ramus éducateur, les procédés scolaires et la nature de la réalité ", Pédagogues et juristes, éd. P. Mesnard, Paris, Vrin, 1963, p. 207-221 (p. 214, visible en ligne, sur l'analyse et synthèse).

21. Dialectique (p. 157, où distinguer veut dire «tirer un trait, séparer les parties, les sections ») : "après que l'enfant aura distingué toute la sentence [tout le syllogisme qui organise Ovide, Heroides, I, v. 47-74], avec cette interprétation diligente, ou qu'il l'aura distinctement apprise de son maître, et de ces 49 <vers> il en aura remarqués pour la proposition [la majeure] 9, pour l'assomption [la mineure] 26, et pour la conclusion 14. Ayant ainsi décrit son syllogisme, non seulement il se représentera les parties d'icelui, mais la sentence des parties.» On est au chapitre 3, "Illustration de l'exercice de l'analyse par exemple de poète " (éd. Dassonville, p. 155-156). La traduction française du passage d'Ovide y fait 49 vers, que Ramus présente en trois blocs séparés chacun par une ligne blanche. - Pour Montaigne au contraire, « distinguer » (tirer un trait, séparer) est évident, et peut se faire sans maître: "J'entends que la matière <de mes chapitres> se distingue soi-même. Elle montre assez où elle se change, où elle conclut, où elle commence, où elle se reprend » (Essais, III, 9, éd. Villey, Paris, PUF, 1965, p. 995).

22. Quand un Richesource en 1681 emploie le vocabulaire de Ramus, "synthèse " et " analyse », le second mot change de sens et a déjà la signification actuelle. Richesource distingue une première rhétorique, qu'il appelle "Synthétique, comme qui dirait faiseuse, composeuse \& ajusteuse ", parce qu'elle « enseigne de quelle manière l'Orateur doit joindre toutes les parties ou pièces d'un discours", et une seconde rhétorique, baptisée "Analytique ", art du critique ou "Critiste » (Les Plaisirs de la lecture aux vives lumières du Camouflet, Paris, à la Renommée, art. XXIII, p. 85-86, cité par Delphine Denis, "Rhétorique et herméneutique: imprécations raciniennes", Exercices de rhétorique, $1 \mid 2013$, note 2).

23. J. Pierrot, Recueil de discours, narrations, lettres, lieux-communs, Paris, L. Hachette, 1831 (avec des discours de ses élèves, dont Sainte-Beuve et Cousin), p.ij: «Un ancien professeur, dont l'Université moderne vénère encore la mémoire et les traditions, M. Gueroult aîné, conseillait aux 
rhétoriciens [aux élèves de la classe de rhétorique] de chercher, à leur début, les premiers secrets de l'art d'écrire dans Lucain et dans Thomas [Antoine-Léonard Thomas, 1732-1785], avant de les demander à Fénélon et à Virgile. »

24. Rome, la ville sans origine. L'Énéide: un grand récit du métissage?, Paris, Le Promeneur / Gallimard, 2011.

\section{AUTEUR}

\section{FRANCIS GOYET}

Univ. Grenoble-Alpes, RARE - Rhétorique de l'Antiquité à la Révolution 